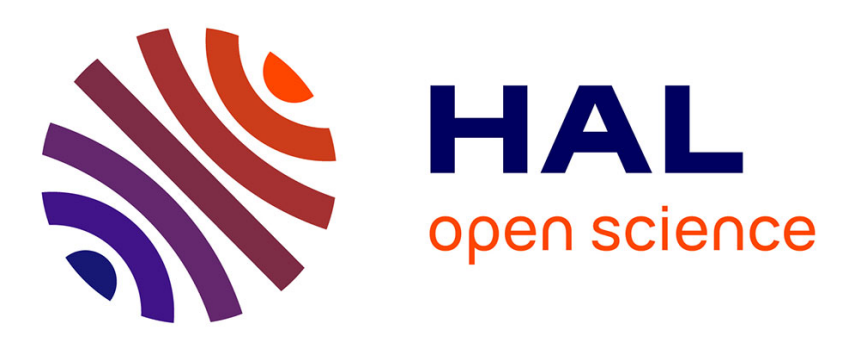

\title{
In vitro activity of beta-lactam antibiotics against CTX-M-producing
}

\author{
M. Tärnberg, A. Östholm-Balkhed, H.-J. Monstein, A. Hällgren, H. \\ Hanberger, L. E. Nilsson
}

\section{- To cite this version:}

M. Tärnberg, Å. Östholm-Balkhed, H.-J. Monstein, A. Hällgren, H. Hanberger, et al.. In vitro activity of beta-lactam antibiotics against CTX-M-producing. European Journal of Clinical Microbiology and Infectious Diseases, 2011, 30 (8), pp.981-987. 10.1007/s10096-011-1183-4 . hal-00666673

\section{HAL Id: hal-00666673 https://hal.science/hal-00666673}

Submitted on 6 Feb 2012

HAL is a multi-disciplinary open access archive for the deposit and dissemination of scientific research documents, whether they are published or not. The documents may come from teaching and research institutions in France or abroad, or from public or private research centers.
L'archive ouverte pluridisciplinaire $\mathbf{H A L}$, est destinée au dépôt et à la diffusion de documents scientifiques de niveau recherche, publiés ou non, émanant des établissements d'enseignement et de recherche français ou étrangers, des laboratoires publics ou privés. 
1 In vitro activity of beta-lactam antibiotics against CTX-M-producing Escherichia coli

2

3 Maria Tärnberg ${ }^{1}$, Åse Östholm Balkhed ${ }^{1,2}$, Hans-Jürg Monstein ${ }^{1,3}$, Anita Hällgren ${ }^{1,2}$, Håkan

4 Hanberger $^{1,2}$ and Lennart E Nilsson ${ }^{1}$

5

$6 \quad{ }^{1}$ Department of Clinical and Experimental Medicine, Faculty of Health Sciences, Linköping

7 University, S-581 85 Linköping, Sweden and ${ }^{2}$ Infectious Diseases and ${ }^{3}$ Clinical Microbiology,

$8 \quad$ Linköping University Hospital, S-581 85 Linköping, Sweden.

9

10 Corresponding author:

11 Maria Tärnberg, Clinical Microbiology, Department of Clinical and Experimental Medicine,

12 Linköping University, S-581 85 Linköping, Sweden. Telephone: +46-10-103 20 97, Fax: +46-

13 10-103 45 96, email: maria.tarnberg@liu.se

14

15 Keywords: Extended-spectrum beta-lactamases, susceptibility, MIC 


\section{Abstract}

Objectives: Beta-lactam antibiotics have been discussed as options for treatment of infections caused by multiresistant ESBL-producing bacteria if the MIC is low. The objective of this study was to investigate the in vitro activity of different beta-lactam antibiotics against CTXM-producing E. coli.

Methods: 198 isolates of E. coli, with ESBL-phenotype were studied. PCR-amplification of CTX-M genes and amplicon sequencing were performed. MICs for amoxicillin-clavulanic acid, aztreonam, cefepime, cefotaxime, ceftazidime, ceftibuten, ertapenem, imipenem, mecillinam, meropenem, piperacillin-tazobactam and temocillin were determined with Etest. Susceptibility was defined according to breakpoints of the EUCAST. MIC 50 and $\mathrm{MIC}_{90}$ were calculated.

Results: Isolates with CTX-M group 9 showed higher susceptibility to the beta-lactam antibiotics tested than isolates belonging to CTX-M group 1. More than $90 \%$ of the isolates belonging to CTX-M group 9 were susceptible to amoxicillin-clavulanic acid, ceftazidime, ceftibuten, piperacillin-tazobactam and temocillin. The susceptibility was high to mecillinam, $91 \%$, regardless of CTX-M group. All isolates were susceptible to imipenem and meropenem and $99 \%$ to ertapenem.

Conclusions: This study shows significant differences in susceptibility to different betalactam antibiotics among the CTX-M-producing E. coli isolates and a significant difference for many antibiotics tested between the CTX-M-producing groups 1 and 9. The good in vitro activity of other beta-lactam antibiotics than carbapenems indicate that clinical studies are warranted to examine the potential role of these beta-lactam antibiotics in treatment of infections caused by multiresistant ESBL-producing E. coli. 

3

\section{Introduction}

Until the early twenty-first century many reports of extended-spectrum beta-lactamase (ESBL) producing Enterobacteriaceae described nosocomial outbreaks due to SHV-producing Klebsiella pneumoniae, with TEM as the other major enzyme group frequently found in Enterobacteriaceae. Today, ESBL-producing Escherichia coli are an increasing problem worldwide, including low-prevalence areas such as Scandinavia [1-6], with CTX-M enzymes most commonly found.

There are now more than 90 different CTX-M type beta-lactamases described [7], and divided into five different clusters reflecting similarity on the amino-acid sequence level; CTX-M group 1, CTX-M group 2, CTX-M group 8, CTX-M group 9 and CTX-M group 25 [8].

When reporting antimicrobial susceptibility of invasive isolates to the European Antimicrobial Resistance Surveillance System (EARSS), ESBL-producing E. coli and Klebsiella spp. have been considered resistant to the whole beta-lactam group except carbapenems [9]. The European Committee on Antimicrobial Susceptibility Testing (EUCAST) previously stated in their Expert Rules that ESBL-producing isolates sensitive for cephalosporins should be regarded as intermediate, and intermediate isolates should be regarded as resistant [10], but as of the end of April 2010 changed their recommendations [11]. Since ESBL-producing E. coli have been considered non-susceptible to all beta-lactam antibiotics except carbapenems for a long time, some laboratories may still report these bacteria as cross-resistant to all penicillins and cephalosporins, without previous determination of minimum inhibitory concentration (MIC). 
Recent studies have shown that inadequate empirical therapy (i.e. oxyimino-cephalosporins or other drugs tested in vitro as resistant) of serious infections is more often likely to occur in patients infected by ESBL-producing $E$. coli, and that mortality in this group is higher compared to controls infected by non-ESBL E. coli [12-16]. Several studies have also shown that the increasing mortality is due the inadequate empirical therapy [17-20]. However, it has been discussed if ESBLs, regardless of MIC, always should be judged as cross resistant to all cephalosporins. Occasionally successful treatments with beta-lactam antibiotics other than carbapenems of infections caused by ESBL-producing bacteria have been reported [21-25].

Thus it is of great value to thoroughly investigate ESBL-producing and in particular CTX-Mproducing, E. coli regarding activity of the beta-lactam antibiotics to increase the treatment options for these infections. In this study we determined the MICs of twelve different betalactam antibiotics in a population of ESBL-producing E. coli from a low-prevalence area.

\section{Material \& methods}

\section{Bacterial isolates}

One hundred ninety eight isolates of $E$. coli, collected during January 2002 until December 2007 at the Clinical Microbiology Laboratory, Linköping University Hospital, Sweden, that possessed phenotypic ESBL-characteristics (as deduced by ESBL Etest with cefotaxime and ceftazidime with and without clavulanic acid, performed by the routine laboratory) were included in this study. Isolates from the same patient with identical antibiogram and sample source were excluded. The isolates were mainly of urinary tract origin $(63 \%)$. Wounds and hygiene screenings accounted for $15 \%$ and $14 \%$, respectively, and blood for $6 \%$. 
PCR-amplifications of CTX-M genes were carried out using modified universal forward and reverse primers under conditions described earlier [26]. PCR-amplicons were then sequenced using the M13 sequence primer and edited and compared as described in detail previously [26], as to subgroup the isolates further into CTX-M group 1, 2, 8, 9 or 25.

\section{MIC-determination}

MIC-determinations for twelve different beta-lactam antibiotics were performed using Etest (BioMerieux, Marcy L'Etoile, France) according to the manufacturer's instructions. E. coli ATCC 25922 was used as a reference strain. The antibiotics tested were amoxicillinclavulanic acid, aztreonam, cefepime, cefotaxime, ceftazidime, ceftibuten, ertapenem, imipenem, mecillinam, meropenem, piperacillin-tazobactam and temocillin.

\section{Breakpoints}

Species related MIC breakpoints of the EUCAST were used to classify isolates as susceptible (S), intermediate or resistant (R) [27]. For temocillin two breakpoints according to the British Society for Antimicrobial Chemotherapy (BSAC) were used, 8 and $32 \mathrm{mg} / \mathrm{L}$ respectively [28]. See table 1 .

\section{Statistics}

Differences in antibiotic susceptibility between CTX-M groups 1 and 9 were analysed with non-parametric statistics, using the Mann-Whitney test and software SPSS v. 15.0. Isolates were grouped as susceptible vs. intermediate and resistant. Results were considered significantly different when $\mathrm{p}<0.05$. 
PCR-amplification and DNA-sequence analysis

119 Of the 198 E. coli isolates with an ESBL-phenotype, 188 isolates (95\%) carried CTX-M

120 genes. One hundred thirty two isolates (67\%) belonged to group 1, 55 isolates (28\%)

121 belonged to group 9, and one isolate belonged to group 2.

Susceptibility testing

Cephalosporins. For ceftibuten there was a highly significant difference in susceptibility between CTX-M group 1 and 9 ( $\mathrm{p}<0.001)$, with $10 \%$ susceptible isolates in group 1 compared to $95 \%$ in group 9 . For ceftazidime a similar difference was observed ( $\mathrm{p}<0.001), 8$ $\%$ susceptible isolates in group 1 compared to $95 \%$ in group 9. For cefepime the overall susceptibility was low, $8 \%$, with a significant difference $(\mathrm{p}=0.030)$ seen in CTX-M group 1 with $3 \%$ susceptible isolates compared to $11 \%$ in group 9. No isolates carrying CTX-M

130 genes were susceptible to cefotaxime (table 1).

131 Penicillins. When applying the high BSAC breakpoint $(32 \mathrm{mg} / \mathrm{L})$ for temocillin all isolates

132 were considered susceptible but the lower breakpoint $(8 \mathrm{mg} / \mathrm{L})$ decreased the susceptibility to $13383 \%$. Using the lower BSAC breakpoint, the difference in temocillin susceptibility between 134 CTX-M group $1(79 \%)$ and group $9(91 \%)$ was statistically significant $(\mathrm{p}=0.048)$. Ninety 135 one percent of all the isolates were susceptible for mecillinam (table 1).

136 Penicillin/beta-lactamase inhibitor combinations. For amoxicillin-clavulanic acid there was a 137 significant difference in susceptibility between CTX-M group 1 and $9(\mathrm{p}=0.014)$, with $80 \%$ 138 susceptible isolates in group 1 compared to $95 \%$ susceptible in group 9 (table 1). Also for 139 piperacillin-tazobactam there was a statistically significant difference in susceptibility 
140 between CTX-M group 1 and group $9(\mathrm{p}=0.009), 82 \%$ and $96 \%$ susceptible isolates,

141 respectively (table 1).

142 Aztreonam. For aztreonam there was a highly significant difference in susceptibility between

143 CTX-M group 1 and 9 ( $\mathrm{p}<0.001)$, with $0 \%$ susceptible isolates in group 1 compared to $18 \%$

144 in group 9 (table 1$)$.

145 Carbapenems. All isolates were susceptible to imipenem and meropenem, $99 \%$ were also

146 susceptible to ertapenem (table 2).

147

\section{Discussion}

149

150 The main findings of this study are the substantial differences in susceptibility to different

151 beta-lactam antibiotics among the studied CTX-M-producing E. coli isolates in general and

152 the big difference in susceptibility between the CTX-M-producing groups 1 and 9 for several

153 antibiotics, in particular.

154

155 To our knowledge this is the most extensive study of the in vitro activity of several different

156 beta-lactam antibiotics against a large collection of CTX-M-producing E. coli. Although

157 ESBL-producing isolates hydrolyzes penicillins and cephalosporins, this study shows that

158 MIC-determination of different beta-lactam antibiotics can be of value in finding treatment

159 options. Comparisons of results with other investigators are often difficult, mainly because of

160 lack of MIC-distributions in the presentations, no presentation of breakpoints used and a

161 different distribution of ESBL-producing enzymes or no specification of the enzymes.

162

163 The MIC-distribution for cefotaxime, ceftazidime and cefepime in the present study is similar

164 to the results in a British study by Livermore et al. [29], and in a Belgian study by Rodriguez- 
Villalobos et al. [30], but when applying the current EUCAST breakpoints on their MIC-

166 distributions the rate of susceptibility differs. For cefotaxime the overall susceptibility rate of

167 the British study is similar to ours (1\%) but for the Belgian MIC-distributions the

168 susceptibility is as high as $14 \%$. For ceftazidime the corresponding figures are $34 \%$

169 susceptible isolates in our study compared to $12 \%$ in the UK-study and only $4 \%$ in the

170 Belgian study. Finally for cefepime the overall susceptibility rate is much higher in both the

171 UK and Belgian studies, $28-34 \%$ compared to only $8 \%$ in our study. Some of these

172 differences may be explained by differences in production of different groups of ESBL-

173 enzymes among the isolates. In this study $65 \%$ of the CTX-M enzymes were group 1 and 28

$174 \%$ group 9 compared to 74\% CTX-M group 1 and only $3 \%$ group 9 in the UK-study and

175 approximately $40 \%$ CTX-M group 1 and $5 \%$ group 9 in the Belgian study. The differences

176 in susceptibility seen between CTX-M group 1 and 9 for ceftazidime (8 and $94 \%$,

177 respectively) are in concordance with a recent Norwegian study [31].

178

179 The MIC-distribution of ceftibuten among the ESBL-producing E. coli in the present study

180 was wide, with MIC-values ranging from 0.125 - >128 mg/L. This has also been shown in a

181 Taiwanese study [32]. We found a big difference between the CTX-M groups with $95 \%$

182 susceptible isolates in the CTX-M group 9 and $10 \%$ in CTX-M group 1.

184 The overall susceptibility for mecillinam in this study is remarkably high, $91 \%$. There is one

185 case report on successful treatment of pyelonephritis caused by a CTX-M-producing E. coli

186 with mecillinam [23], but the value of treatment of UTIs caused by ESBL-producing

187 organisms with mecillinam needs to be further evaluated. Thomas et al. advice against use of

188 mecillinam in serious infections due to an inoculum effect seen in vitro [33]. 
Treatment of lower urinary tract infections caused by CTX-M-producing E. coli is often

191 limited to nitrofurantoin and fosfomycin due to frequent co-resistance to other oral preparations such as quinolones, trimethoprim and trimethoprim-sulfamethoxazole. During

193 pregnancy and childhood treatment with fluoroquinolones is not recommended. In this

194 perspective the possibility to treat with an oral beta-lactam agent is an option and the results

195 in this study indicate good in-vitro activity for mecillinam and for ceftibuten among isolates

196 in CTX-M group 9.

197

198 Temocillin is a penicillin only commercially available in Belgium and the UK, which has

199 been showing promising effect in vitro on ESBL-producing isolates [30, 34]. Clinical studies

200 on the efficacy of temocillin in treatment of infections caused by ESBL-producing isolates are

201 scarce. However, there is a case series of severe sepsis caused by ESBL-producing isolates, 202 showing promising results with temocillin [25]. In our study the overall susceptibility for 203 temocillin was high, 83 or $100 \%$, depending on the breakpoint ( 8 or $32 \mathrm{mg} / \mathrm{L}$ ) used. These 204 susceptibility results similar to Rodriguez-Villalobos et al. (81 and 99\%, respectively) [30], 205 but differs from Livermore et al. (64 and >99\%, respectively) [34].

The overall susceptibility for amoxicillin-clavulanic acid was the same as for piperacillintazobactam (84\%), and as high as $94 \%$ for amoxicillin-clavulanic acid and $96 \%$ for piperacillin-tazobactam for the strains belonging to CTX-M group 9.

211 The amoxicillin-clavulanic acid results in our study are similar to those from Sorlozano et al.

212 [35], but differs from others [36-38]. Amoxicillin-clavulanic acid has been used for successful 213 treatment of cystitis caused by multidrug resistant CTX-M 15 producing E. coli [22], but 214 failed in 1 of 11 bloodstream infections [17]. 
216 In the study by Rodriguez-Villalobos et al. [30], $82 \%$ of the ESBL-producing E. coli were 217 susceptible to piperacillin-tazobactam applying the EUCAST breakpoints. Using CLSI

218 breakpoints (susceptible $\leq 16 \mathrm{mg} / \mathrm{L}$ ), several studies show moderate to high activity (69-95\%)

219 for piperacillin-tazobactam [35, 36, 39, 40]. Successful treatment with piperacillin-tazobactam 220 of infections caused by ESBL-producing bacteria has also been reported [41]. According to 221 Peterson even severe infections should be considered treatable when the MIC value imply so 222 [42], but most authors advise against the use of piperacillin-tazobactam in ESBL infections.

224 The overall susceptibility rate for aztreonam was extremely low (8\%) and for strains 225 belonging to CTX-M group 1 none of the strains were susceptible. The differences seen 226 between CTX-M group 1 and 9 for aztreonam are in concordance with a recent Norwegian 227 study [31].

The susceptibility for carbapenems was very high $(99-100 \%)$ and in agreement with worldwide studies $[30,32,35,39,43,44]$. All isolates were susceptible to imipenem and 231 meropenem and only two isolates were resistant to ertapenem. Carbapenems are still a recommended therapy against invasive infections caused by ESBL-producing bacteria.

234 In conclusion this study shows significant difference in susceptibility to different beta-lactam 235 antibiotics among CTX-M-producing E. coli. Isolates with ESBL enzymes belonging to CTX236 M group 9 were in general more susceptible to these antibiotics than group 1. Further comparative studies using different methods for MIC determination (such as Etest, agar and 238 broth dilutions) are needed to confirm the good in vitro activity of beta-lactam antibiotics 239 other than carbapenems, against CTX-M-producing E. coli, as demonstrated in this study. 
Studies of different isolates with MICs close to the breakpoints with time killing curve

241 experiments and animal in vivo experiments are also needed. However, most warranted are

242 clinical studies to examine the potential role of these beta-lactam antibiotics in the treatment

243 of infections caused by multiresistant ESBL-producing E. coli.

245 Acknowledgement

246 This study was presented as a poster at the $19^{\text {th }}$ European Congress of Clinical Microbiology

247 and Infectious Diseases in Helsinki, Finland, 16-19 May 2009.

\section{Funding}

250 This work was supported by the Medical Research Council of Southeast Sweden.

\section{Transparency declaration}

253 Nothing to declare.

\section{Authors' contributions}

256 MT, ÄÖB, HJM, AH, HH and LEN participated in the conception, design, drafting of the manuscript, and final approval of the version to be published. MT was responsible for the

258 laboratory work, analysis, statistical calculations and interpretations. MT, ÅÖB and LEN 259 were responsible for the clinical strain collection.

\section{References}


263 1. Alsterlund, R., et al., Multiresistant CTX-M-15 ESBL-producing Escherichia coli in

264

265

266

267

268

269

270

271

272

273

274

275

276

277

278

279

280

281

282

283

284

285

286

287

288

289

290

291

292

293

294

295

296

297

298

299

300

301

302

303

304

305

306

307

308 southern Sweden: Description of an outbreak. Scand J Infect Dis, 2009: p. 1-6.

2. $\quad$ Fang, H., et al., Molecular epidemiology of extended-spectrum beta-lactamases among Escherichia coli isolates collected in a Swedish hospital and its associated health care facilities from 2001 to 2006. J Clin Microbiol, 2008. 46(2): p. 707-12.

3. Kjerulf, A., et al., The prevalence of ESBL-producing E. coli and Klebsiella strains in the Copenhagen area of Denmark. Apmis, 2008. 116(2): p. 118-24.

4. $\quad$ Lytsy, B., et al., The first major extended-spectrum beta-lactamase outbreak in Scandinavia was caused by clonal spread of a multiresistant Klebsiella pneumoniae producing CTX-M-15. Apmis, 2008. 116(4): p. 302-8.

5. Naseer, U., et al., Nosocomial outbreak of CTX-M-15-producing E. coli in Norway. Apmis, 2007. 115(2): p. 120-6.

6. Nyberg, S.D., et al., Detection and molecular genetics of extended-spectrum betalactamases among cefuroxime-resistant Escherichia coli and Klebsiella spp. isolates from Finland, 2002-2004. Scand J Infect Dis, 2007. 39(5): p. 417-24.

7. $\quad$ http://www.lahey.org/Studies/other.asp\#table1, George Jacoby \& Karen Bush. Last date accessed 9 August 2010.

8. Bonnet, R., Growing group of extended-spectrum beta-lactamases: the CTX-M enzymes. Antimicrob Agents Chemother, 2004. 48(1): p. 1-14.

9. http://www.rivm.nl/earss/Images/Earss\%20manual2005_tcm61-21261.pdf, EARSS Manual 2005., European Antimicrobial Resistance Surveillance System. Last date accessed 9 August 2010.

10.

http://www.srga.org/eucastwt/EUCAST\%20Expert\%20rules\%20final\%20April 20080407.pdf, Expert Rules in Antimicrobial Susceptibility Testing, The European Committee on Antimicrobial Susceptibility Testing (EUCAST). Last date accessed 9 August 2010.

11. http://www.eucast.org/expert_rules/, The European Committe on Antimicrobial Susceptibility Testing, Last date accessed 9 August 2010.

12. Melzer, M. and I. Petersen, Mortality following bacteraemic infection caused by extended spectrum beta-lactamase (ESBL) producing E. coli compared to non-ESBL producing E. coli. J Infect, 2007. 55(3): p. 254-9.

13. Schwaber, M.J. and Y. Carmeli, Mortality and delay in effective therapy associated with extended-spectrum beta-lactamase production in Enterobacteriaceae bacteraemia: a systematic review and meta-analysis. J Antimicrob Chemother, 2007. 60(5): p. 913-20.

14. Cordery, R.J., et al., Evaluation of risk factors for the acquisition of bloodstream infections with extended-spectrum beta-lactamase-producing Escherichia coli and Klebsiella species in the intensive care unit; antibiotic management and clinical outcome. J Hosp Infect, 2008. 68(2): p. 108-15.

15. Gudiol, C., et al., Bacteraemia due to extended-spectrum \{beta\}-lactamase-producing Escherichia coli (ESBL-EC) in cancer patients: clinical features, risk factors, molecular epidemiology and outcome. J Antimicrob Chemother, 2009.

16. Ortega, M., et al., Analysis of 4758 Escherichia coli bacteraemia episodes: predictive factors for isolation of an antibiotic-resistant strain and their impact on the outcome. $\mathrm{J}$ Antimicrob Chemother, 2009. 63(3): p. 568-74. 
17. Rodriguez-Bano, J., et al., Bacteremia due to extended-spectrum beta-lactamaseproducing Escherichia coli in the CTX-M era: a new clinical challenge. Clin Infect

18. Tumbarello, M., et al., Predictors of mortality in patients with bloodstream infections caused by extended-spectrum-beta-lactamase-producing Enterobacteriaceae: importance of inadequate initial antimicrobial treatment. Antimicrob Agents Chemother, 2007. 51(6): p. 1987-94.

19. Tumbarello, M., et al., Bloodstream infections caused by extended-spectrum-betalactamase- producing Escherichia coli: risk factors for inadequate initial antimicrobial therapy. Antimicrob Agents Chemother, 2008. 52(9): p. 3244-52.

20. Song, K.H., et al., Clinical outcomes of spontaneous bacterial peritonitis due to extended-spectrum beta-lactamase-producing Escherichia coli and Klebsiella species: a retrospective matched case-control study. BMC Infect Dis, 2009. 9: p. 41.

21. Bin, C., et al., Outcome of cephalosporin treatment of bacteremia due to CTX-M-type extended-spectrum beta-lactamase-producing Escherichia coli. Diagn Microbiol Infect Dis, 2006. 56(4): p. 351-7.

22. Lagace-Wiens, P.R., et al., Treatment of lower urinary tract infection caused by multidrug-resistant extended-spectrum-beta-lactamase-producing Escherichia coli with amoxicillin/clavulanate: case report and characterization of the isolate. $\mathrm{J}$ Antimicrob Chemother, 2006. 57(6): p. 1262-3.

23. Nicolle, L.E. and M.R. Mulvey, Successful treatment of ctx-m ESBL producing Escherichia coli relapsing pyelonephritis with long term pivmecillinam. Scand J Infect Dis, 2007. 39(8): p. 748-9.

24. Rodriguez-Bano, J., et al., Community infections caused by extended-spectrum betalactamase-producing Escherichia coli. Arch Intern Med, 2008. 168(17): p. 1897-902.

25. Gupta, N.D., R.E. Smith, and I. Balakrishnan, Clinical efficacy of temocillin. J Antimicrob Chemother, 2009. 64(2): p. 431-3.

26. Monstein, H.J., M. Tarnberg, and L.E. Nilsson, Molecular identification of CTX-M and blaOXY/K1 beta-lactamase genes in Enterobacteriaceae by sequencing of universal M13-sequence tagged PCR-amplicons. BMC Infect Dis, 2009. 9: p. 7.

http://www.eucast.org/fileadmin/src/media/PDFs/EUCAST_files/Disk_test_docu ments/EUCAST_breakpoints_v1.1.pdf, Breakpoint tables for interpretation of MICs and zone diameters., The European Committe on Antimicrobial Susceptibility Testing, Last date accessed 9 August 2010.

28. http://www.bsac.org.uk/db/_documents/Version_8_-_January_2009.pdf, BSAC methods for antimicrobial susceptibility testing, British Society for Antimicrobial Chemotherapy. Last date accessed 8 April 2010.

29. Livermore, D.M., et al., Orthodox and unorthodox clavulanate combinations against extended-spectrum beta-lactamase producers. Clin Microbiol Infect, 2008. 14 Suppl 1: p. 189-93.

30. Rodriguez-Villalobos, H., et al., In vitro activity of temocillin against extended spectrum beta-lactamase-producing Escherichia coli. J Antimicrob Chemother, 2006. 57(4): p. 771-4.

31. Tofteland, S., et al., Effects of phenotype and genotype on methods for detection of extended-spectrum-beta-lactamase-producing clinical isolates of Escherichia coli and Klebsiella pneumoniae in Norway. J Clin Microbiol, 2007. 45(1): p. 199-205.

32. Liao, C.H., et al., In vitro activities of 16 antimicrobial agents against clinical isolates of extended-spectrum beta-lactamase-producing Escherichia coli and Klebsiella 
pneumoniae in two regional hospitals in Taiwan. J Microbiol Immunol Infect, 2006. 39(1): p. 59-66.

33. Thomas, K., et al., Activity of mecillinam against ESBL producers in vitro. $\mathbf{J}$

34. Livermore, D.M., et al., Activity of temocillin against prevalent ESBL-and AmpCproducing Enterobacteriaceae from south-east England. J Antimicrob Chemother, 2006. 57(5): p. 1012-4.

35. Sorlozano, A., et al., Activity in vitro of twelve antibiotics against clinical isolates of extended-spectrum beta-lactamase producing Escherichia coli. J Basic Microbiol, 2007. 47(5): p. 413-6.

36. Hoban, D.J., et al., In vitro activity of tigecycline against 6792 Gram-negative and Gram-positive clinical isolates from the global Tigecycline Evaluation and Surveillance Trial (TEST Program, 2004). Diagn Microbiol Infect Dis, 2005. 52(3): p. 215-27.

37. Oteo, J., et al., Spread of Escherichia coli strains with high-level cefotaxime and ceftazidime resistance between the community, long-term care facilities, and hospital institutions. J Clin Microbiol, 2006. 44(7): p. 2359-66.

38. Prakash, V., et al., Oral and parenteral therapeutic options for outpatient urinary infections caused by enterobacteriaceae producing CTX-M extended-spectrum betalactamases. Antimicrob Agents Chemother, 2009. 53(3): p. 1278-80.

39. Colodner, R., et al., First national surveillance of susceptibility of extended-spectrum beta-lactamase-producing Escherichia coli and Klebsiella spp. to antimicrobials in Israel. Diagn Microbiol Infect Dis, 2007. 57(2): p. 201-5.

40. Sader, H.S., et al., Comparative activities of cefepime and piperacillin/tazobactam tested against a global collection of Escherichia coli and Klebsiella spp. with an ESBL phenotype. Diagn Microbiol Infect Dis, 2007. 57(3): p. 341-4.

41. Gavin, P.J., et al., Clinical correlation of the CLSI susceptibility breakpoint for piperacillin-tazobactam against extended-spectrum-beta-lactamase-producing Escherichia coli and Klebsiella species. Antimicrob Agents Chemother, 2006. 50(6): p. 2244-7.

42. Peterson, L.R., Antibiotic policy and prescribing strategies for therapy of extendedspectrum beta-lactamase-producing Enterobacteriaceae: the role of piperacillintazobactam. Clin Microbiol Infect, 2008. 14 Suppl 1: p. 181-4.

43. Hernandez, J.R., et al., Comparative in vitro activity of ertapenem against extendedspectrum beta-lactamase-producing Escherichia coli and Klebsiella pneumoniae isolated in Spain. Int J Antimicrob Agents, 2006. 28(5): p. 457-9.

44. Kiremitci, A., et al., In vitro activity of ertapenem and other carbapenems against extended-spectrum beta-lactamase producing Escherichia coli and Klebsiella pneumoniae clinical isolates in a tertiary care center in Turkey. Expert Opin Pharmacother, 2008. 9(9): p. 1441-9. 
399 Table 1. MIC-distributions. Breakpoints according to a) BSAC and b) EUCAST ( $\leq / \mathrm{R}>$ ). $\mathrm{MIC}_{50}$ in bold underlined, MIC 90 in bold italics.

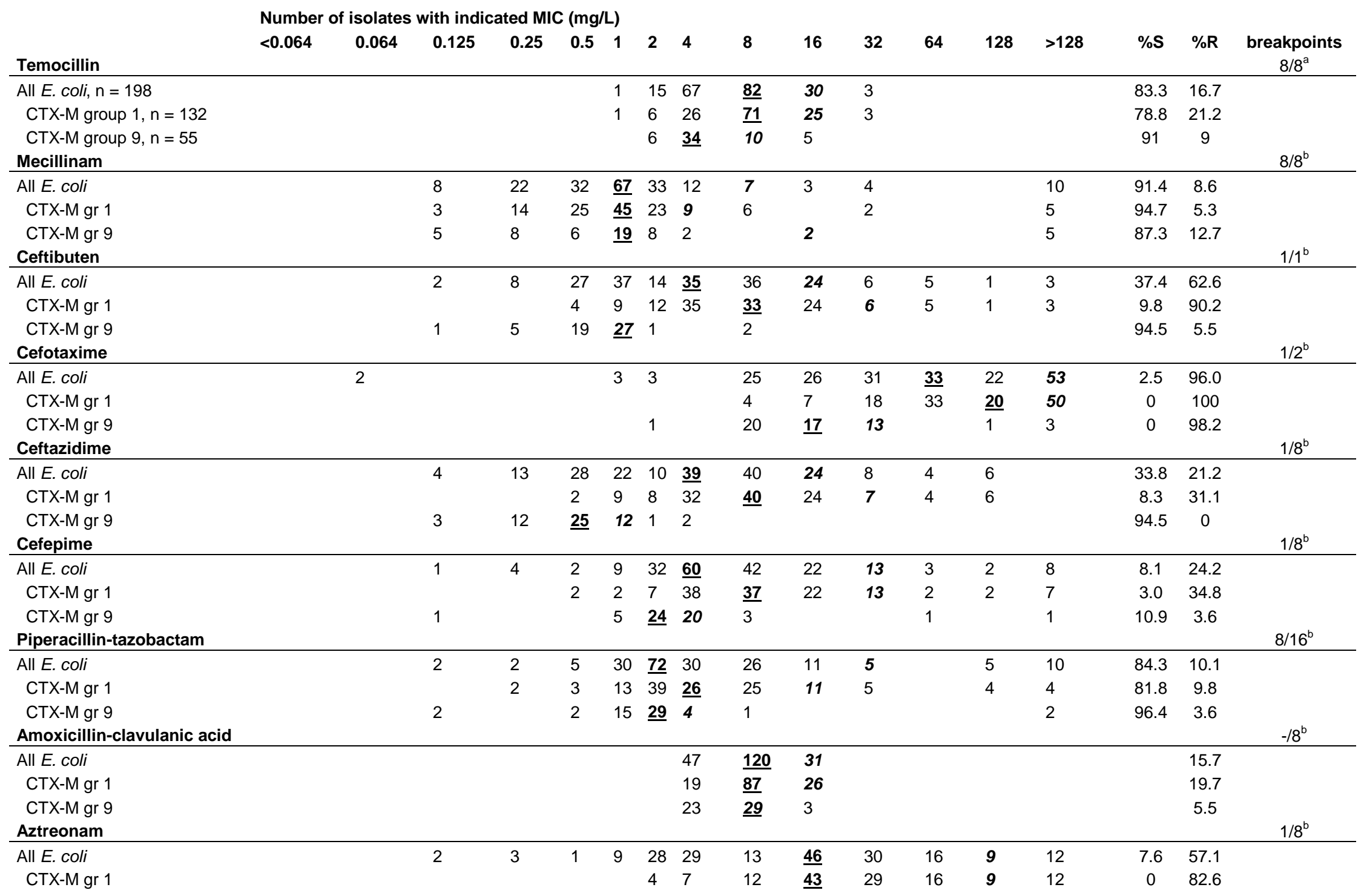


401 Table 2. MIC-distributions. Breakpoints according to EUCAST $(\mathrm{S} \leq / \mathrm{R}>)$. $\mathrm{MIC}_{50}$ in bold underlined, $\mathrm{MIC}_{90}$ in bold italics.

\begin{tabular}{|c|c|c|c|c|c|c|c|c|c|c|c|c|c|c|c|c|c|}
\hline Imipenem & \multicolumn{17}{|c|}{ Number of isolates with indicated MIC (mg/L) } \\
\hline All E. coli $\mathrm{n}=198$ & & & & & & 28 & 161 & 8 & 1 & & & & & & 100 & 0 & \\
\hline CTX-M group $9 \mathrm{n}=55$ & & & & & & 5 & $\underline{47}$ & 2 & 1 & & & & & & 100 & 0 & \\
\hline Meropenem & & & & & & & & & & & & & & & & & $2 / 8$ \\
\hline All E. coli & & 1 & 33 & $\underline{127}$ & 29 & 6 & 2 & & & & & & & & 100 & 0 & \\
\hline CTX-M gr 1 & & & 11 & $\underline{87}$ & 28 & 6 & & & & & & & & & 100 & 0 & \\
\hline All E. coli & & 10 & 52 & $\underline{52}$ & 46 & 16 & 8 & 11 & 1 & 2 & & & & & 98.5 & 1.0 & \\
\hline CTX-M gr 1 & & 4 & 21 & $\overline{34}$ & $\underline{38}$ & 15 & 8 & 11 & 1 & & & & & & 99.2 & 0 & \\
\hline CTX-M gr 9 & & 3 & $\underline{30}$ & 15 & $\overline{5}$ & & & & & 2 & & & & & 96.4 & 3.6 & \\
\hline
\end{tabular}

Number of isolates with indicated MIC $(\mathrm{mg} / \mathrm{L})$ 